\title{
Comparative analysis on Imbalanced Multi-class Classification for Malware Samples using CNN
}

\author{
Arwa Alzammam ${ }^{1}$, Hamad Binsalleeh ${ }^{1}$, Basil AsSdhan ${ }^{2}$, \\ Konstantinos G. Kyriakopoulos ${ }^{3}$, Sangarapillai Lambotharan ${ }^{3}$ \\ ${ }^{1}$ Department of Computer Science, Imam Muhammad Ibn Saud Islamic University, Riyadh, Saudi Arabia \\ ${ }^{2}$ Department of Electrical Engineering, King Saud University, Riyadh, Saudi Arabia \\ ${ }^{3}$ Wolfson Schol of Mechanical, Electrical and Manufactoring Engineering, Loughborough University, Loughborough, UK \\ e-mail: asalzammam@sm.imamu.edu.sa,hmbinsalleeh@imamu.edu.sa,bsadhan@ksu.edu.sa, \\ K.Kyriakopoulos@lboro.ac.uk, S.Lambotharan@lboro.ac.uk
}

\begin{abstract}
Malware considered as one of the main actors in cyber attacks. Everyday, the number of unique malware samples are in the rise, however the ratio of benign software still greatly outnumbers malware samples. In machine learning, such datasets are known as imbalanced, where the majority class label greatly dominate the other ones. In this paper, we present a comparative analysis and evaluation of some of the proposed techniques in the literature to address the problem of classifying imbalanced multiclass malware datasets. We used Convolutional Neural Network (CNN) as a classification algorithm to study the effect of imbalanced datasets on deep learning approaches. The experiments are conducted on three publicly available imbalanced datasets. Our performance analysis shows that methods such as cost sensitive learning, oversampling and cross validation have positive effects on the model classification performance with varying degree. While others like using pre-trained models require more special parameter settings. However, best practice may change according to the problem domain.
\end{abstract}

Index Terms-Malware Classification, Imbalanced Dataset, Deep learning

\section{INTRODUCTION}

Malware is a malicious software that is designed to intentionally performs harmful actions on computer systems. The level of damage differs based on the intention of the malware designer and the nature of the targeted systems. The number of malware samples are increasing day by day. For instance, it was reported that around 350,000 new malware samples are registered every day in the year of 2018 [1]. Despite the growing rate of malware every day, antivirus vendors are still heavily dependent on signature-based detection system built by handcrafting patterns from malware codes or malware behavior to detect malware instances. Such a method faces difficulties to detect zero-day or simple polymorphic malware that employs any obfuscation mechanism. This problem pronounced the need to come up with automated techniques to detect malware instances. Anomaly detection mechanisms help in closing this gap by capturing behavioral features that can be utilized in the generation of automated signatures.

This work was supported in part by the Gulf Science,Innovation and Knowledge Economy Programme of theU.K. Government under UK-Gulf Institutional Link Grant IL279339985
Machine learning models are used to differentiate between benign and malicious, or categorize malicious behaviors based on the extracted features from a dataset [2]. The performance of machine learning techniques rely on the quality of the provided datasets in representing the problem to be solved. One of the challenges when applying machine learning techniques on malware detection is the lack of large sized labeled data of malware instances [3]. Malware datasets suffer from the imbalanced dataset problem, where the majority class label greatly dominate the other ones [4]. The problem of imbalanced dataset has been studied in the literature [4] and many techniques were proposed to minimize its effect on the performance of machine learning algorithms [5]. However, with the evolution of machine learning algorithms, the problem of imbalanced datasets remains one of the challenging issues to be resolved [2]. For instance, researchers spend considerable amount of time trying to determine the most appropriate technique to adopt and reduce the effects of imbalanced dataset problem [6]. In the literature, there is a lack of systematic analysis on the use of different practices to resolve the problem of imbalance multi-class classification using deep learning algorithms [6].

In this paper, we present a comparative analysis and evaluation of some of the proposed techniques in the literature to address the problem of classifying imbalanced multiclass malware datasets. We leverage the success of utilizing Convolutional Neural Network (CNN) classification algorithm in classifying malware samples to different malware family classes. The evaluation is based on the two main techniques that are used to tackle the problem of imbalanced datasets: data sampling, and algorithmic enforcement techniques. We conduct our experiments on three publicly available malware datasets. The rest of the paper is structured as follows: Section II provides a background on malware classification. Section III shows the datasets used in this paper. Section IV explains the experimental evaluation setup. Section V provides an analysis of the performance of the different methods used to enhance the performance for multi-class imbalanced dataset classification. Finally, conclusions and future work are provided in 
Section VI.

\section{Malware Classification}

\section{A. Deep Learning and Malware Classification}

Various neural network architectures such as Convolutional Neural Network (CNN) [7] and Recurrent Neural Network (RNN) [8] are used for malware classification. However, the difference lies in the data pre-processing of malware files to be analyzed. For example, CNN usually performs better on images of malware samples [9]. While malware textual representation fed to RNN [10] for better classification performance. $\mathrm{CNN}$ is a feed forward neural network with three layers, convolutional layer, pooling layer, and fully connected layer. The convolutional layer is the core layer of the $\mathrm{CNN}$, where all the calculations of feature vectors and learning process are performed. Pooling layer is used to reduce the size of representations and dimensionality of data. In the fully connected layer, all of the previous node's outputs connected to produce a dimensional vector of the same size as the labelled classes [10]. Recently, malware classification problem adopted the use of CNN algorithm by feeding malware binaries as an image files such as the work in [9], [4] and [7]. In [11] they find that malware instances belonging to the same malware family share the same image structure. This observation enable the use of image representation of malware binaries to recognize variants of the same malware family. For instance, many contributions in the literature used $\mathrm{CNN}$ for malware classification using gray-scale images of malware binaries such as [9] in which they build three different convolutional neural network architectures to classify malware families using grayscale images of malware binaries. They use the open-source malware dataset provided by Microsoft during the Big Data Innovation Gathering [12].

\section{B. Imbalanced Dataset Classification}

A considerable amount of time is spent on pre-processing datasets to build good machine learning models. The problem of imbalanced dataset is considered as one of the issues to be resolved before conducting any machine learning exercise. For instance, classifier models tend to be biased towards the majority class when an imbalanced dataset is used [5]. In the literature, many techniques has been proposed to minimize the effect of imbalanced datasets on the performance of classification algorithms. In [5] they categorize the methods of dealing with imbalanced datasets into two levels: firstly, datalevel approaches such as data sampling methods (e.g. oversampling and under-sampling) and feature selection methods. Secondly, algorithm-level approaches such as cost sensitive methods and hybrid methods.

1) Data-level approaches: such as data sampling which is considered as the most straightforward method to deal with imbalance classification problem [6]. There are two main strategies to re-sample a dataset: under-sampling, or oversampling. Both techniques manipulate the number of samples in each class by increasing or decreasing the samples to reach equal representation for all classes. Under-sampling techniques randomly eliminate samples from the dominating classes until reaching the size of the least represented class. However, the use of this technique may result in deleting the most representative samples which may negatively affect the performance of the classifier [5]. On the other hand, over-sampling techniques randomly duplicate samples in the least represented classes until reaching the size of the most represented class. However, this technique may cause an over fitting in the classification model [2].

2) Algorithm-level approaches: such as cost sensitive learning which is a method used at the classifier level to improve the performance of classifying imbalanced datasets [6]. According to [16] cost sensitive learning can be classified into two major types: 1) build cost sensitive classifiers. 2) convert a classifier to be cost sensitive which also called meta-learning. The meta-learning method consists of two types: thresholding and sampling. Calculating class weights in loss function is of type "sampling". The idea of weighting is to assign higher weights to the less represented classes which will also have a higher miss-classification cost [17]. It's also said that calculating class weights is equivalent to over-sampling [16].

Softmax activation function paired with categorical crossentropy loss in Keras are used in this experiment for multiclass classification with class weights. The formula for the weighted softmax loss is [4]:

$$
J_{0}=-\frac{1}{M} \sum_{i=0}^{m} \sum_{i=0}^{k} w_{k} * 1(y(i)=j) \log P_{j}^{i}
$$

Where $w_{k}$ is the class weight and calculated by using the scikit-learn method compute_class_weight. The calculated class weights are added to the class weights parameter in Keras "fit" method and is applicable during training periods only.

Another approach is the hybrid approach where both datalevel and algorithm-level methods are applied by using a pretraind model such as VGG16, VGG19 and Xception [18] [19]. They are also a popular approach for training a relatively small dataset such as Malimg and Microsoft datasets. Pre-trained CNN models are trained on a very large Image dataset such as ImageNet [20] and are available on Keras as applications used for feature extraction, fine tuning or prediction [21]. Since malware datasets are small sized with training set around 10,000 samples. There are multiple works classifying malware on pre-trained models such as [22] and [4]. The work in [22], classified images of malware family using CNN based on VGG-16 and train their model on two different datasets Microsoft and Malimg. Using pre-trained models for training small dataset provides advantages however, they are trained on specific vision tasks and datasets. Using them on a different classification task with a different type of dataset enforce a challenge. To tackle this challenge the work in [4] modified the softmax loss to a weighted loss and fine-tuned the model on the pre-trained VGG19. The weights are determined according to class weights in the dataset. In this part of the experiment we examine this approach. We fine tune VGG-19 to test the positive effects acquired from using pre-trained model for 
TABLE I

MalWARE IMAGE ClassificATION RESEARCHES

\begin{tabular}{|c||c|c|c|c|c|}
\hline Work & Dataset & Classifier & Practices & Evaluation metrics provided & Features \\
\hline \hline$[11]$ & Malimg & k-nearest neighbors & Cross validation & Accuracy: 98\% & GIST \\
\hline$[4]$ & Malimg & CNN & $\begin{array}{l}\text { Weighted softmax } \\
\text { Pretrained model }\end{array}$ & $\begin{array}{l}\text { Loss: } 98.63 \% \\
\text { VGG + } \\
\text { CNN generated features }\end{array}$ \\
\hline$[9]$ & Microsoft & CNN & N.A & $\begin{array}{l}\text { Accuracy: 99.76\% } \\
\text { Loss: 0.0231 } \\
\text { Confusion matrix }\end{array}$ & CNN generated features \\
\hline$[13]$ & IoTPOT & CNN & Cross validation (hold-out) & Accuracy: 93.17\% & CNN generated features \\
\hline$[14]$ & Malimg & CNN & N.A & $\begin{array}{l}\text { Accuracy: 94\% } \\
\text { Confusion matrix }\end{array}$ & $\begin{array}{l}\text { LBP + } \\
\text { CNN generated features }\end{array}$ \\
\hline$[15]$ & Malimg and Malheur & KNN, SVM & N.A & Accuracy: 98.84\%, 98.88\% & Wavelet transforms \\
\hline
\end{tabular}

small dataset [18]. We use the pre-trained model for two tasks: 1) bottleneck features extraction and 2) fine tuning with and without class weights. Since we are using Keras API, instead of modifying the loss function we take advantage of the class weight parameter called when training the model using "fit" method. Which performs the same functionality of the weighted loss. The idea behind it is to apply weight for each class according to their representation statue within the dataset when calculating the categorical cross-entropy loss [21].

3) Other approaches: such as employing cross validation (CV) mechanism which is widespread in machine learning and deep learning fields. Several works in intrusion detection systems applied cross validation in deep learning such as [11], [23], [13], [24]. However, using cross validation may cause biases in the model depending on the domain [25]. Concept drift and the similarities between malware families place malware classification in one of the domains that becomes positively bias when using cross validation according to [23]. However, using $\mathrm{CV}$ provides a better performance than a baseline in any problems [25]. In this experiment a cross validation applied with 10 folds, 50 epochs each. The best performing round are taken as the representative model for this approach.

\section{Evaluation Metrics}

The most used metric to evaluate a classifier for malware classification problem is accuracy. However, there are other metrics in the literature that can provide more information for the evaluation. For instance, the accuracy measure has some limitations in evaluating the performance for classifying imbalanced multi-class datasets. In the following, we give a brief description of the used metrics:

1) Accuracy: it measures the ability of the classifier to recognize the correct class, which is defined in terms of True Positive (TP), True Negative (TN), False Positive (FP) and False Negative (FN) as follows:

$$
\text { Accuracy }=\frac{T P+T N}{T P+T N+F P+F N}
$$

TABLE II

Datasets Size And Class Distributions

\begin{tabular}{|l||c|c|l|}
\hline Dataset & Size & Classes & \multicolumn{1}{|c|}{ Class Distributions } \\
\hline Malimg & 9233 & 24 & {$[122-116-2949-1591-$} \\
& & & $198-200-146-177-$ \\
& & & $142-381-431-213-$ \\
& & & $184-123-159-136-$ \\
& & & $162-158-80-128-$ \\
& & & $132-408-97-800]$ \\
\hline Microsoft & 10868 & 9 & {$[1013-2942-398-2478-$} \\
& & & $1228-1541-42-751-$ \\
& & & $475]$ \\
\hline VirusTotal & 17205 & 12 & {$[1309-584-2288-1726-$} \\
& & & $1142-788-2290-1795-$ \\
& & & $1191-1319-2209-564]$ \\
\hline
\end{tabular}

2) Precision: it measures the classifier's ability to not to classify negative samples as positive:

$$
\text { Precision }=\frac{T P}{T P+F P}
$$

3) Recall: it measures the classifier's ability to classify positive samples positively:

$$
\text { Recall }=\frac{T P}{T P+F N}
$$

4) F1-score: it is considered as the harmonic mean between precision and recall:

$$
\text { F1score }=\frac{2 * \text { precision } * \text { recall }}{\text { precision }+ \text { recall }}
$$

\section{Dataset Specification}

We select three different publicly available malware datasets to present fair and comparable performance evaluation. The datasets vary in the number of malware families, samples per family and type of families. In the following, we provide a brief description of each malware dataset. Table I shows some of the research papers that use the selected datasets. We highlights the type of classification algorithm, important configurations, evaluation metrics, and applied techniques to resolve the problem of imbalanced dataset. Table II shows the size, number of malware families, and number of samples in each family for each of the selected datasets. All datasets are 


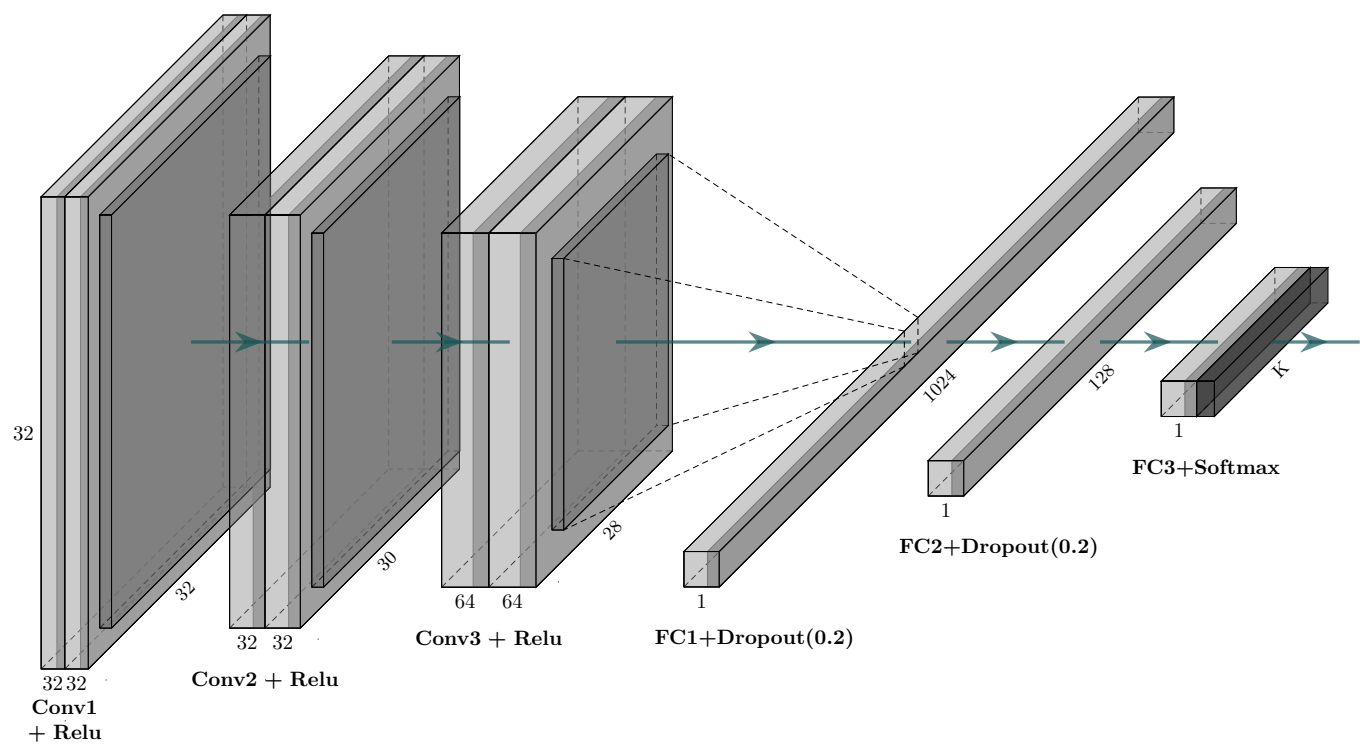

Fig. 1. CNN Model Architecture

considered as imbalanced with a total number of 45 family classes.

\section{A. Malimg Dataset}

Malimg dataset contains gray-scale images of malware samples [11]. Several research papers use this dataset to classify malware using CNN such as [11], [4], [14] and [15]. The malware samples are of the following types: Worm, PWS, Trojan, Dialer, TrojanDownloader and Rogue.

\section{B. Microsoft Dataset}

Microsoft dataset is considered as the standard benchmark for malware classification and has been cited in more than 50 research papers according to [12]. The dataset contains malware samples each sample has two files: .asm and .byte [12]. To use the dataset for CNN we convert the hexadecimal representation file for each malware to grayscale images according the guidelines suggested by [11]. The malware samples are of the following types: Worm, Adware, Backdoor, Trojan, TrojanDownloader, and any kind of obfuscated malware.

\section{VirusTotal Dataset}

The third dataset is collected from VirusTotal [26]. VirusTotal is a public resource providing access to malware samples and other functionalities such as analyzing suspicious files, URLs, search for IP addresses, domains or file hashes. To label malware samples according to their family name, JSON file was generated for each malware sample and then processed using the AVClass malware labeling Tool [27]. The dataset is collected over one month from 2018. It is important to note that malware images of this dataset are generated by converting malware EXE to gray-scale images of a fixed width of size 256 and belong to the following malware types: Adware, Trojan, Virus, Ransomware and Worm.

\section{EXPERIMENTAL EVALUATION SETUP}

We conduct a set of experiments to demonstrate the effectiveness of different techniques at tackling the problem of imbalanced multi-class malware datasets. In our experiments, we used $\mathrm{CNN}$ as a classification algorithm to classify malware instances into families. Figure 1 presents the $\mathrm{CNN}$ architecture which is considered as the basic and the commonly used in the literature [13] [28]. The CNN architecture consists of three convolutional layers with Relu function [29] as the activation function. Each convolutional layer is followed by a pooling layer and finally two fully connected layer. Softmax [30] is used as the activation function for the last fully connected layer. The experiments are conducted using Keras API with TensorFlow back-end [21]. We can summarize the the steps of our experiments as follows:

1) Convert malware binaries to gray-scale images with the size of $32 \times 32$.

2) Perform the classification using the $\mathrm{CNN}$ for each imbalanced dataset without using any of the techniques to reduce the effects of imbalanced nature.

3) Apply each one of the mentioned techniques in Section II on the three different datasets separately.

4) Evaluate the performance of the CNN models based on the evaluation metrics in Section II

\section{Performance Evaluation}

We elaborate the outcome of different experiments to demonstrate the effect of applying different practices mentioned in Section II that are used to enhance the performance of classifying imbalanced multi-class malware datasets. In Table III, we summarize the performance evaluation for eight (8) main conducted experiments using the evaluation metrics 
TABLE III

PERFormance EVALUATION

\begin{tabular}{|c|c|c|c|c|c|c|c|c|c|c|c|c|c|c|c|}
\hline & \multicolumn{5}{|c|}{ Malimg } & \multicolumn{5}{|c|}{ Microsoft } & \multicolumn{5}{|c|}{ VirusTotal } \\
\hline$\#$ & Acc\% & Pre\% & Rec\% & F1\% & loss & Acc\% & Pre\% & Rec\% & F1\% & loss & Acc\% & Pre\% & Rec\% & F1\% & loss \\
\hline 1 & 96.15 & 99.99 & 99.99 & 99.99 & 20.97 & 91.16 & 76.65 & 83.25 & 79.81 & 59.75 & 87.96 & 84.98 & 89.23 & 87.05 & 72.14 \\
\hline 2 & 96.31 & 99.99 & 99.99 & 99.99 & 19.51 & 92.36 & 80.64 & 83.73 & 82.15 & 47.33 & 88.05 & 89.68 & 86.92 & 88.28 & 72.71 \\
\hline 3 & 96.63 & 99.99 & 99.99 & 99.99 & 21.73 & 94.56 & 83.48 & 90.09 & 86.66 & 41.47 & 89.26 & 77.98 & 94.65 & 85.51 & 63.69 \\
\hline 4 & 88.54 & 99.99 & 99.99 & 99.99 & 60.16 & 81.81 & 79.99 & 33.33 & 47.05 & 72.50 & 80.87 & 83.99 & 78.35 & 81.08 & 106.36 \\
\hline 5 & 99.94 & 99.99 & 99.99 & 99.99 & 0.23 & 98.31 & 95.43 & 98.65 & 97.01 & 13.64 & 96.06 & 96.72 & 95.82 & 96.27 & 23.37 \\
\hline 6 & 88.25 & 99.99 & 94.73 & 97.29 & 43.14 & 89.09 & 78.57 & 73.68 & 76.04 & 35.49 & 66.14 & 77.45 & 51.53 & 61.89 & 102.66 \\
\hline 7 & 88.25 & 99.99 & 94.73 & 97.29 & 43.52 & 89.28 & 79.16 & 72.72 & 75.81 & 36.14 & 65.73 & 78.16 & 52.30 & 62.67 & 103.40 \\
\hline 8 & 78.06 & 92.30 & 99.99 & 95.99 & 54.01 & 90.87 & 85.57 & 85.14 & 85.35 & 27.47 & 88.00 & 85.82 & 83.52 & 84.66 & 38.47 \\
\hline
\end{tabular}

in Section II. In the following, we discuss the evaluation details of the experiments:

1) Baseline: This is considered as the baseline where there is no treatments for the imbalanced dataset problem. The same model configurations used for the rest of experiments.

2) Class weights: With the addition of class weights as described in Section II, there is a slight increase in performance for all three datasets. The improvement is more evident in the Microsoft dataset with an increase in the accuracy by $1.2 \%$, F1-score by $2.34 \%$ and decrease the loss by $12.42 \%$. The VirusTotal dataset shows noticeable improvement which contribute to the increase of the precision by $4.86 \%$. However, the recall has been decreased by $2.31 \%$. This may be due to the added weights to the minority classes over majority classes, which results in the classifier being affected by other data factors. Thus, any majority class with no significant features may have been affected by being misclassified. For example, in the per class recall performance measurement for the baseline, the majority class such as Gepys and Hematite have 459 and 320 samples with 0.99 and 0.91 recall. However, when class weights are employed the recall decreases to 0.53 and 0.48 respectively. In conclusion, the overall performance for the class weights is still better than the baseline according to the average of all evaluation metrics.

3) Cross validation: It shows a positive effect in terms of accuracy and loss compared to the baseline. However, there is a positive bias that comes with cross validation and the fact that the increased performance is not for all evaluation measures. Moreover, cross validation takes at least one sample from each family during the training process, which contributes to the increase of the performance of accuracy. However, there is a possibility that the classifier may not be able to handle zero-day malware classification as stated in [23].

4) Under sampling: Due to the small sized datasets, the model over-fitted during the training of this experiment for all datasets. Another factor may have contributed is the large number of epochs $(=50)$, which is considered unnecessary for small sized datasets. As a result, we can conclude that using under sampling as a practice on its own to deal with imbalanced multi-class for malware image classification problem is not beneficial.

5) Over sampling: Over sampling for all datasets shows the best performance across all evaluation metrics.

6) Pre-trained model without class weights: Since the VGG19 model is trained on a different classification task, it performed worse than the baseline.

7) Pre-trained model with class weights: The Microsoft and VirusTotal datasets show consistency in their performance when adding class weights. In which their performance increase compared to the previous experiment. It remains however less than the baseline performance for both datasets. As for Malimg dataset, only loss is affected when adding class weights in which it decreases in performance by $0.36 \%$.

8) Bottleneck features: Both Microsoft and VirusTotal datasets benefited from bottleneck features. While it has a negative effect on the Malimg dataset. Seeing the varying positive and negative reactions in the last three experiments indicate that the practice of using pre-trained model requires complexity (e.g. special parameter settings and a preprocess step where other data factors are considered) and should be considered carefully when applied to malware classification.

The work in [31] provided a theoretical study on the impact of data imbalanced in relation to other data factors. They suggested that before trying imbalance recovery methods to deal with imbalanced datasets. It is important to consider other factors that may affect the classification performance degradation such as noise, overlapping, and small disjuncts.

In our case it is evident that there exist other factors that may cause classification performance degradation on the imbalanced dataset more than its imbalanced nature. One of the factors is the data noise, which affects the overall performance of VirusTotal dataset. Although it has more samples per class in comparison to the other two datasets, the conversion to images comes from raw binary file (exe files) and not from the hexadecimal representations of raw data. In Table IV, we present Imbalance Ratio (IR) [32] and Shannon's entropy (H) [33] for the all datasets. Based on IR VirusTotal dataset is shown to be the least imbalanced dataset. However, the results of $\mathrm{H}$ disagree with IR rating, making the Malimg as the least imbalanced dataset. IR takes the highest majority class and 
lowest minority class only. The difference is more pronounced in Malimg than VirusTotal with $\{2949: 80\}$ and $\{2290: 564\}$ respectively. It shows that it's not a suitable measure for multiclass.

TABLE IV

IMBALANCE RATIO

\begin{tabular}{|c||c|c|c|}
\hline Imbalance Factor & Malimg & Microsoft & VirusTotal \\
\hline IR & 36.8625 & 70.047 & 4.060 \\
H & -420.44 & -2856.15 & -2813.04 \\
\hline
\end{tabular}

\section{CONClusions And Future Work}

In this paper we provide a comparative analysis to tackle the problem of Imbalanced multi-class classification by using practices known in the literature to enhance the performance of classifying imbalanced dataset. Our evaluation concluded that the use of over-sampling outperforms other techniques. However, there is a need to use specific-domain features to improve the performance of classifying imbalanced malware datasets. As a future work, we plan to study the problem on a domain-specific level by applying tuning based extracted features from malware samples to see the positive effects imposed on the classification problem. We also want to study the imbalance factor and data factors that may cause the performance degradation and its relation to the domain by calculating the Imbalance Degree (ID) and Likelihood Ratio (LRID) presented in [34] and [35].

\section{REFERENCES}

[1] AV-TEST, "The AV-TEST Security Report 2017/18," Magdeburg, Germany, 2018, accessed 2019-05-22. [Online]. Available: https://www.av-test.org/en/about-the-institute/publications/

[2] O. E. David and N. S. Netanyahu, "Deepsign: Deep learning for automatic malware signature generation and classification," 2015 International Joint Conference on Neural Networks (IJCNN), Jul 2015.

[3] D. Ucci, L. Aniello, and R. Baldoni, "Survey of machine learning techniques for malware analysis," Computers \& Security, vol. 81, p. 123-147, Mar 2019.

[4] S. Yue, "Imbalanced malware images classification: a cnn based approach," arXiv preprint arXiv:1708.08042, Aug 2017.

[5] J. L. Leevy, T. M. Khoshgoftaar, R. A. Bauder, and N. Seliya, "A survey on addressing high-class imbalance in big data," Journal of Big Data, vol. 5, no. 1, p. 42, 2018.

[6] M. Buda, A. Maki, and M. A. Mazurowski, "A systematic study of the class imbalance problem in convolutional neural networks," Neural Networks, vol. 106, p. 249-259, Oct 2018.

[7] W. Wang, M. Zhu, X. Zeng, X. Ye, and Y. Sheng, "Malware traffic classification using convolutional neural network for representation learning," in 2017 International Conference on Information Networking (ICOIN). IEEE, 2017, pp. 712-717.

[8] R. Pascanu, J. W. Stokes, H. Sanossian, M. Marinescu, and A. Thomas, "Malware classification with recurrent networks," in 2015 IEEE International Conference on Acoustics, Speech and Signal Processing (ICASSP). IEEE, 2015, pp. 1916-1920.

[9] D. Gibert, "Convolutional neural networks for malware classification," University Rovira i Virgili, Tarragona, Spain, 2016.

[10] L. Deng, D. Yu et al., "Deep learning: methods and applications," Foundations and Trends ${ }^{\circledR}$ in Signal Processing, vol. 7, no. 3-4, pp. 197-387, 2014.

[11] L. Nataraj, S. Karthikeyan, G. Jacob, and B. Manjunath, "Malware images: visualization and automatic classification," in Proceedings of the 8 th international symposium on visualization for cyber security. ACM, 2011, p. 4.
[12] R. Ronen, M. Radu, C. Feuerstein, E. Yom-Tov, and M. Ahmadi, "Microsoft malware classification challenge," CoRR, vol. abs/1802.10135, Aug 2018.

[13] J. Su, V. D. Vasconcellos, S. Prasad, S. Daniele, Y. Feng, and K. Sakurai, "Lightweight classification of iot malware based on image recognition," in 2018 IEEE 42nd Annual Computer Software and Applications Conference (COMPSAC), vol. 2. IEEE, 2018, pp. 664-669.

[14] J.-S. Luo and D. C.-T. Lo, "Binary malware image classification using machine learning with local binary pattern," in 2017 IEEE International Conference on Big Data (Big Data). IEEE, 2017, pp. 4664-4667.

[15] A. Makandar and A. Patrot, "Malware class recognition using image processing techniques," in 2017 International Conference on Data Management, Analytics and Innovation (ICDMAI). IEEE, 2017, pp. 76-80.

[16] C. X. Ling and V. S. Sheng, "Cost-sensitive learning and the class imbalance problem," Encyclopedia of Machine Learning: Springer, pp. $231-235,2008$

[17] K. M. Ting, "Inducing cost-sensitive trees via instance weighting," in European Symposium on Principles of Data Mining and Knowledge Discovery. Springer, 1998, pp. 139-147.

[18] K. Simonyan and A. Zisserman, "Very deep convolutional networks for large-scale image recognition," arXiv preprint arXiv:1409.1556, p. 14, 2014.

[19] F. Chollet, "Xception: Deep learning with depthwise separable convolutions," in Proceedings of the IEEE conference on computer vision and pattern recognition, 2017, pp. 1251-1258.

[20] J. Deng, W. Dong, R. Socher, L.-J. Li, K. Li, and L. Fei-Fei, "Imagenet: A large-scale hierarchical image database," in 2009 IEEE conference on computer vision and pattern recognition. Ieee, 2009, pp. 248-255.

[21] F. Chollet, "Keras Documentation," Keras.Io, 2015. [Online]. Available: https://keras.io/

[22] M. Kalash, M. Rochan, N. Mohammed, N. D. Bruce, Y. Wang, and F. Iqbal, "Malware classification with deep convolutional neural networks," in 2018 9th IFIP International Conference on New Technologies, Mobility and Security (NTMS). IEEE, 2018, pp. 1-5.

[23] F. Pendlebury, F. Pierazzi, R. Jordaney, J. Kinder, and L. Cavallaro, "Tesseract: Eliminating experimental bias in malware classification across space and time," arXiv preprint arXiv:1807.07838, 2018

[24] C. Curtsinger, B. Livshits, B. G. Zorn, and C. Seifert, "Zozzle: Fast and precise in-browser javascript malware detection." in USENIX Security Symposium. San Francisco, 2011, pp. 33-48.

[25] C. Schaffer, "Selecting a classification method by cross-validation," Machine Learning, vol. 13, no. 1, pp. 135-143, 1993.

[26] "Virustotal," 2018, accessed 2019-05-22. [Online]. Available: https://www.virustotal.com

[27] M. Sebastián, R. Rivera, P. Kotzias, and J. Caballero, "Avclass: A tool for massive malware labeling," in International Symposium on Research in Attacks, Intrusions, and Defenses. Springer, 2016, pp. 230-253.

[28] G. E. Dahl, J. W. Stokes, L. Deng, and D. Yu, "Large-scale malware classification using random projections and neural networks," in 2013 IEEE International Conference on Acoustics, Speech and Signal Processing. IEEE, 2013, pp. 3422-3426.

[29] V. Nair and G. E. Hinton, "Rectified linear units improve restricted boltzmann machines," in Proceedings of the 27th international conference on machine learning (ICML-10), 2010, pp. 807-814.

[30] J. S. Bridle, "Probabilistic interpretation of feedforward classification network outputs, with relationships to statistical pattern recognition," in Neurocomputing. Springer, 1990, pp. 227-236.

[31] Y. Lu, Y.-m. Cheung, and Y. Y. Tang, "Bayes imbalance impact index: A measure of class imbalanced dataset for classification problem," arXiv preprint arXiv:1901.10173, 2019.

[32] V. García, J. S. Sánchez, and R. A. Mollineda, "On the effectiveness of preprocessing methods when dealing with different levels of class imbalance," Knowledge-Based Systems, vol. 25, no. 1, pp. 13-21, 2012.

[33] A. Rényi et al., "On measures of entropy and information," in Proceedings of the Fourth Berkeley Symposium on Mathematical Statistics and Probability, Volume 1: Contributions to the Theory of Statistics. The Regents of the University of California, 1961.

[34] J. Ortigosa-Hernández, I. Inza, and J. A. Lozano, "Measuring the classimbalance extent of multi-class problems," Pattern Recognition Letters, vol. 98, pp. 32-38, 2017.

[35] R. Zhu, Z. Wang, Z. Ma, G. Wang, and J.-H. Xue, "Lrid: A new metric of multi-class imbalance degree based on likelihood-ratio test," Pattern Recognition Letters, vol. 116, pp. 36-42, 2018. 